\title{
Research on resilience capacity assessment of urban water supply system in China under flood and drought disaster
}

Hui Zhao

Qingdao University of Technology

Zhijie Li ( $\square$ corresponding.lizhijie0815@126.com )

Qingdao University of Technology

Jinning Liu

Qingdao University of Technology

Jingqi Zhang

Qingdao University of Technology

Zhiguo Shao

Qingdao University of Technology

\section{Research Article}

Keywords: flood and drought disaster, urban water supply system, index system, resilience capacity, cloud model

Posted Date: July 28th, 2021

DOl: https://doi.org/10.21203/rs.3.rs-747478/v1

License: (1) This work is licensed under a Creative Commons Attribution 4.0 International License.

Read Full License 
1 Research on resilience capacity assessment of urban water supply

2 system in China under flood and drought disaster

3 Hui Zhao ${ }^{1}$, Zhijie Li ${ }^{1, *}$, Jinning Liu ${ }^{1,+}$, Jingqi Zhang ${ }^{1,+}$, Zhiguo Shao $^{1,2,+}$

$4{ }^{1}$ School of management engineering, Qingdao University of Technology, Qingdao, 266520, China

$5 \quad{ }^{2}$ Tongji University Sustainable Development and New Urbanization Think Tank, Shanghai 200092, China

$6 \quad{ }^{*}$ corresponding.lizhijie0815@126.com

7 theseauthorscontributedequallytothiswork

$8 \quad$ Highlights

- The evaluation index system of urban water supply system is further improved, and the indexes are qualitatively classified and quantitatively analyzed.

- The cloud model is used to simulate the four indexes of the study case to ensure the reliability of the simulation.

- The whole water supply system is added as one of the toughness evaluation indexes to ensure the integrity of evaluation.

- The toughness of urban water supply system was evaluated and compared by two methods.

- According to the four system dimensions of urban water supply system, the paper puts forward the targeted promotion strategy.

\section{Abstract}

Under the influence of global climate change, urban flood and drought disasters occur frequently, so that is extremely important to construct the resilience capacity of urban water supply system. Based on the framework of system toughness and capability analysis, using correlation analysis and factor analysis to construct the index system of resilience capacity assessment of urban water supply system, which reflects the four dimensions of water source, water plants, water supply and distribution network and users, and five dimensions of social, natural environment, economy, physics and organization, and the weights of all indexes are given. The multi-level comprehensive evaluation model based on cloud model and the index comprehensive evaluation index method based on entropy weight were used to evaluate the resilience of the water supply system in Qingdao under flood and drought disasters, and the evaluation results of the two methods were compared. Finally, based on the evaluation results, the influencing factors of water supply system resilience were analyzed, and the corresponding strategies to improve the resilience of water supply system were proposed, in order to provide decision support for the planning and construction of urban water supply system resilience in the near and long term. 
Keywords: flood and drought disaster; urban water supply system; index system; resilience capacity; cloud model

\section{Introduction}

32 For the past few years, the global climatic variation is severe, and extreme weather events occur frequently. The frequency and impact degree of urban flood and drought disasters is gradually increasing, and the urban water supply system is facing more severe challenges ${ }^{[1]}$. Especially in China, on August 16, 2011, high temperatures occurred in eastern Sichuan, Chongqing, most of Hubei, northeastern Guizhou and most of the south of the Yangtze River. Eighty-seven counties (cities and districts) in Guizhou Province were affected by the drought to varying degrees, and more than 20 million people affected by the drought were facing drinking water difficulties. On July 21, 2012, heavy rain hit most parts of China, with Beijing and its surrounding areas hit by the heaviest rainstorms in 61 years. As a result of the torrential rains, 62 counties (cities and districts) in Beijing, Tianjin and Hebei provinces (municipalities directly under the central government) suffered floods, affecting 5.4 million people, affecting 530 thousand hectares of crops, collapsing 30,000 houses, damaging 50 reservoirs, 3,427 dikes with 1,032 kilometers of water, 2,565 bank embankments, and 1,053 sluices. The rainstorm in Fangshan District alone damaged 200 kilometers of water supply pipelines, resulting in a direct economic loss of 33.1 billion RMB. In the flood season of 2016, the cumulative rainfall in Qingdao, Yantai, Weifang and Weihai in Jiaodong region was 30 50\% less than that in the same period of the year. In addition, affected by the drought in the previous three years, the underground water level continued to decline, and the reservoirs generally lacked water. As of September 30, the total volume of large and medium-sized reservoirs in the four cities of Jiaodong was 653 million cubic meters, $54.6 \%$ less than that in the same period of the year. There were 21 large and medium-sized reservoirs below the dry or dead water level, accounting for $95 \%$ of the total number of dry reservoirs in the province. In July, Qingdao and Weifang were once 107,000 cubic meters short of daily water supply, and 150 million cubic meters short of daily water supply, which seriously affected normal urban water use. In late August 2018, affected by typhoons "Capricorn" and "Vimbiya", many places in Weifang. Shandong Province suffered from torrential rain and flood disasters rarely seen in history, causing serious damage to water supply and power facilities. Thus, in the event of sudden disaster, the urban water supply system becomes extremely fragile.

The failure of the urban water supply system not only affects the normal production and life of the city, but also may lead to the collapse of the entire city operation ${ }^{[2]}$. With the continuous acceleration of China's urbanization, how to effectively ensure the safety of urban water supply and improve the resilience of the water supply system has become an instant problem to be solved.Therefore, this article from the perspective of urban water supply system under inundation and drought resilience, for urban water supply system, based on the correlation analysis and factor analysis to build resilience ability evaluation system for urban water supply system, the multi-level evaluation model based on cloud model of urban water supply system resilience ability are analyzed in the simulation, It is expected to provide theoretical support for 
toughness evaluation of water supply and distribution system in different cities. The toughness capacity assessment

61 framework of urban water supply system constructed of this research is shown in Figure 1.

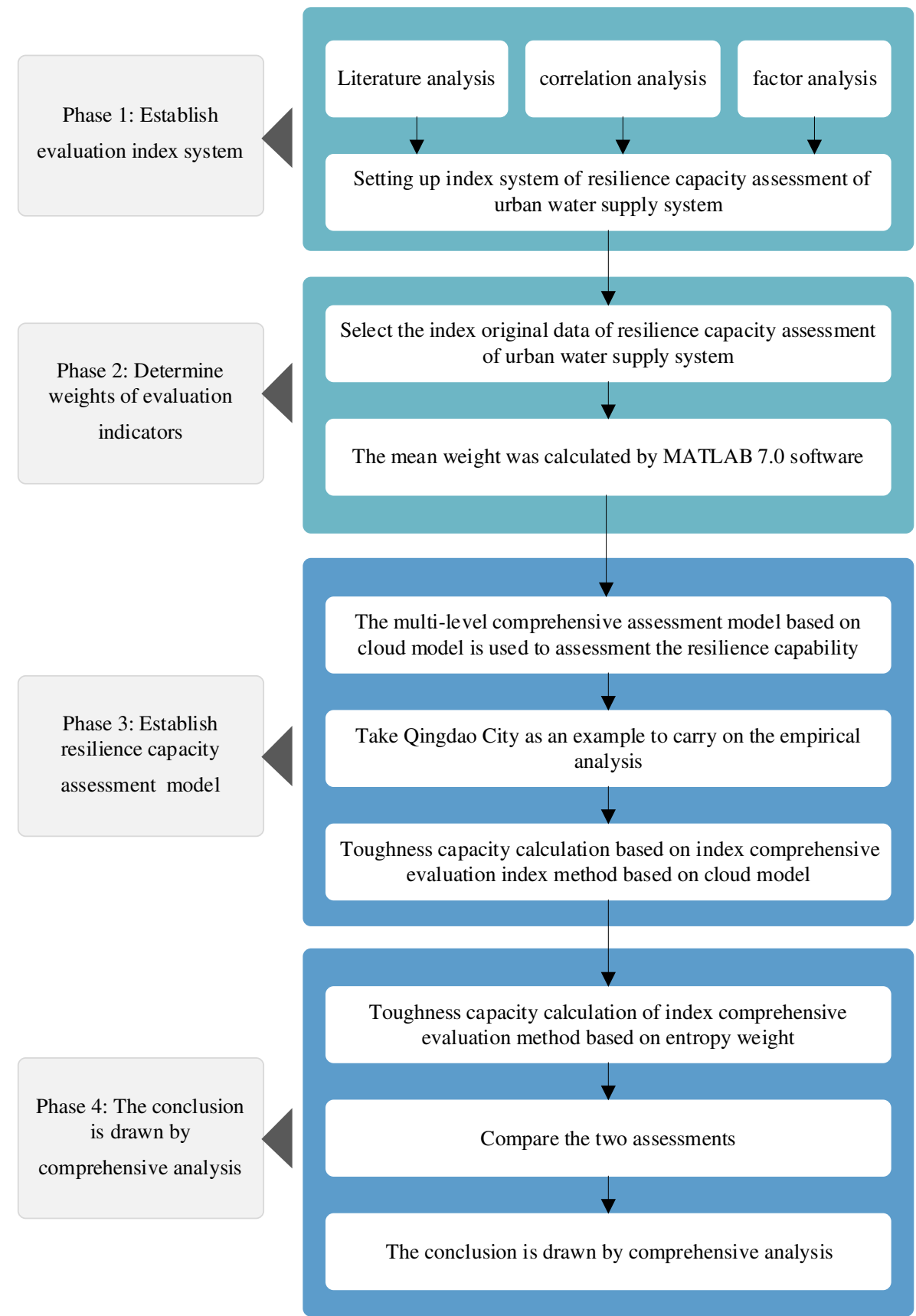

Figure 1. Toughness capacity assessment framework of urban water supply system(From top to bottom, the figure shows the logical process and methods of toughness evaluation of urban water supply system in four parts)

65

66 67

\section{8}

65

\section{Research status of resilience capacity assessment of urban water supply system}

The toughness of urban water supply system has been concerned by more and more scholars at home and abroad. The categories are summarized below.

\section{- The research of foreign scholars}

Fiering $^{[3]}$, Hashimoto ${ }^{[4]}$ et al. studied the water source toughness in urban water supply system earlier, and evaluated the 
water source toughness in urban water supply system with a mathematical model. The model was easy to understand, but

71 the actual case was difficult to apply or the application progress was slow. $\mathrm{Yu}^{[5]}$ et al. established a set of toughness evaluation system for water resources system and classified the toughness of the water resources system with fuzzy clustering method. The index system was comprehensive, but the system resilience was not considered. Tanner ${ }^{[6]}$ et al. evaluated water resource resilience from the perspective of social policy, but the indicators were not quantitative and did not consider the resilience of urban ecological environment system. Simonovic ${ }^{[7]}$ and $\operatorname{Jim}^{[8]}$ et al. studied the water toughness and evaluated the water toughness with a mathematical model. Based on layer theory, Kong ${ }^{[9]}$ et al. used network analysis method to dynamically evaluate the interdependent toughness of urban water supply network, communication network and power network. Pandit and Crittenden ${ }^{[10]}$ summarized the six network attributes of the urban water supply system, and proposed a new water supply system resilience index based on the network topology of the water supply system, and used the analytic hierarchy process to assign weights to the six network attributes. Rehak ${ }^{[1-12]}$ et al. designed the CIERA framework for the toughness assessment of key infrastructure elements, which carried out nonlinear aggregation of point, line and plane infrastructure elements to effectively identify the weak links of the system and put forward targeted measures and suggestions to increase the strength and toughness of system elements.

Zhao Sixiang ${ }^{[13]}$ et al. studied the recovery strategy of the water supply system under sudden water pollution events and established the recovery strategy optimization and selection model. The study showed that reasonable strategy optimization and selection could effectively shorten the emergency recovery time and improve the recovery ability of the water supply system. $\mathrm{Xu} \mathrm{Tao}{ }^{[14]}$ et al. established a grey box model based on the concept and connotation of urban waterlogging resilience, and built an evaluation system of urban waterlogging resilience including three dimensions of resistance, resilience and adaptability with the help of principal component analysis, and evaluated the waterlogging resilience of 238 prefecture-level and above cities in China. Liu Jian and Huang Wenjie ${ }^{[15]}$ constructed an simple index system for the resilience of urban water supply system, which reflected the absorptive capacity, adaptive capacity and resilience of the system, and conducted a visual evaluation of the resilience of the water supply and distribution system using GIS. Yu Kongjian $^{[16]}$ et al. described the application of the concept of elasticity in the field of urban water system, and commented on the evaluation methods and elasticity strategies of water system elasticity. Li Qian ${ }^{[17]}$ et al. put forward that the meaning of seismic toughness of water supply system should include seismic safety and post-earthquake resilience based on the previous research results on seismic safety and post-earthquake resilience of water supply system. The evaluation method is also presented from the perspectives of earthquake safety and post-earthquake recovery capacity, and the evaluation model framework of comprehensive multiple evaluation indexes is proposed. It is pointed out that the seismic toughness evaluation standard of water supply system should match the requirements of the current seismic codes and fortification 
standards.

\section{- Literature Visualization Analysis}

CiteSpace 5.7.R2 software was used to conduct a visual analysis of the literature related to urban water supply system in the recent 10 years, and the results were shown in Figure 2. The study found that,urban water supply system usually includes subsystems such as water source, water plants, water supply and distribution pipe network and users. However, at present, there are many researches on toughness evaluation of water source system and water supply and distribution pipe network system in urban water supply system, and a series of research achievements have been made to enrich the connotation of toughness of water supply and distribution system. However, research often focuses on a certain subsystem in the water supply system, and there are few studies on the construction of the resilience index system and the resilience assessment of the entire water supply system under flood and drought disasters.

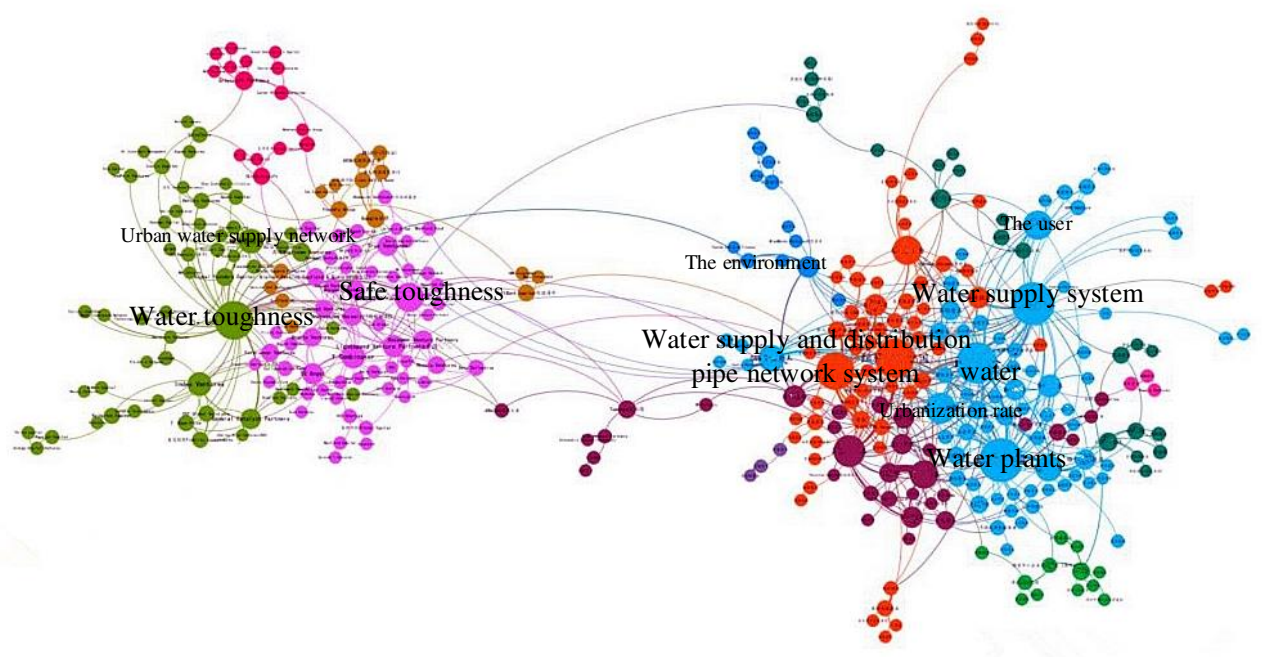

Figure 2. Visual analysis diagram of literature related to urban water supply system(The figure shows a visual analysis of literature in the last 10 years, with foreign countries on the left and China on the right)

\section{Index system of resilience capacity assessment of urban water supply system}

This paper improves the resilience assessment framework of urban water supply systems constructed by Balaei ${ }^{[18]}$ and Lukuba $^{[19]}$ et al., and applies it to the assessment of resilience capacity of urban water supply systems in China(This is shown in Figure 1). On the basis of considering the whole process management of urban water supply system (water source, water plants, water supply and distribution pipe network system and user), fully consider the minimum necessary associated urban management systems closely related to urban water supply system (as shown in Figure 3), such as social, natural environment, economic, physical, organizational and other systems.The toughness capacity evaluation index system of urban water supply system has been further improved.

Therefore, this paper argues that, the toughness capacity of water supply and distribution system is defined as the ability of the water supply and distribution system to withstand disasters, reduce disaster losses, and reasonably allocate resources to 
quickly recover normal water supply from disasters.

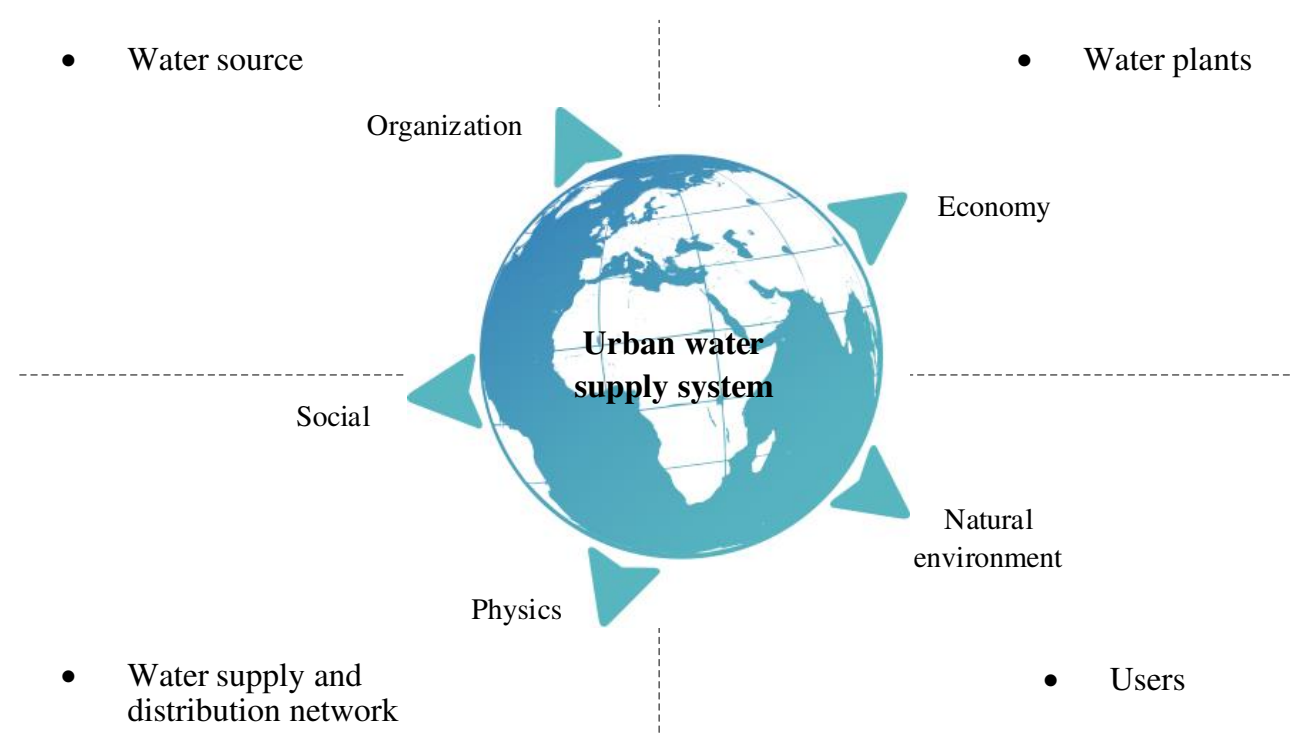

Figure 3. urban water supply systems(The figure shows four dimensions of whole-process water supply system and five dimensions of urban management system)

\section{- Principles and methods of index selection}

According to the principles of comparability, representativeness, feasibility, relevance, non-reproducibility and conformance to toughness implication, the toughness evaluation indexes of urban water supply system were selected ${ }^{[20]}$. Based on the above principles, an open "cylinder index selection model" was established in this paper, as showed in Figure 4. After layers of filtration, toughness indexes of urban water supply system were selected.

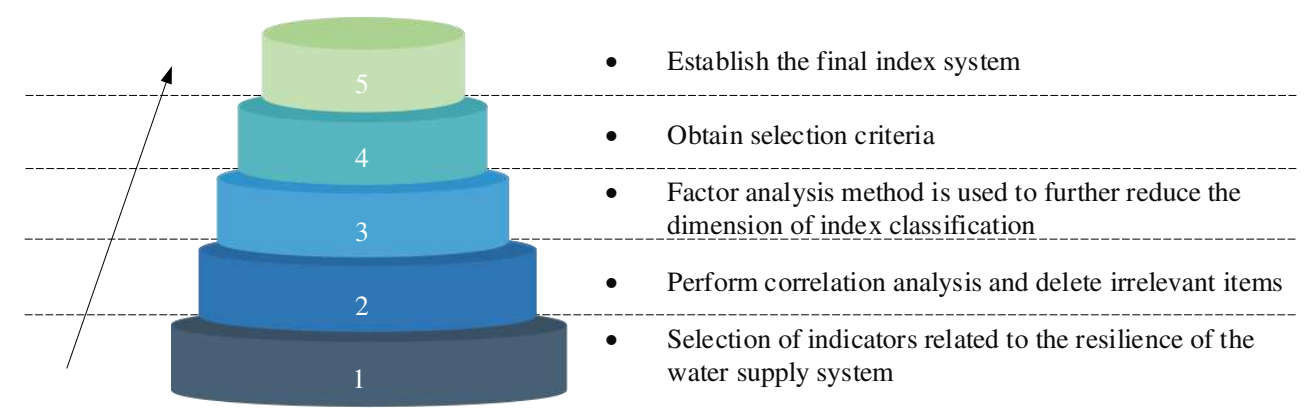

Figure 4. Selection model of toughness index of urban water supply system(According to the arrow direction, from bottom to top, show the establishment process of toughness index selection model of urban water supply system)

\section{Basic data preparation -- audition}

According to the above selection model and selection principles for toughness indicators, all the indicators related to the toughness of the water supply system, including the water source, water supply and water consumption, can be included in the alternative index system. However, due to various reasons, some index data of the selection are unavailable or discontinuous. For example, the degree coefficient of process equipment at the water plant is not available. The net value and original value data of fixed assets of Tianjin, Xinjiang and Gansu are not available in China Urban Water Supply Yearbook, so it is impossible to calculate the degree coefficient of process equipment.Therefore, some of the indicators in 
the audition should be selected first, and those indicators whose data are unavailable or discontinuous should be eliminated.

\section{Index correlation analysis}

So as to ensure the scientific nature, rationality and representativeness of the indicators, sample data from provincial capitals of 31 provinces and municipalities in China from 2014 to 2019 were selected, and 48 indicators were retained based on feasibility and continuity. According to the research background of the problem, considering that the threat of flood and drought disasters will also have an impact on the resilience of the water supply and distribution system, two indicators of flood and drought disaster substitution attribute are added to the above indicators, namely, the amount of reservoir storage at the year-end and the quantity of people affected by flood and drought. Indicators and data sources: "National Hydrological Statistics Annual Report", "Statistical Yearbook of China's Urban Construction", "National Bureau of Statistics", "Provincial Water Resources Bulletin", relevant references.

By calculating the Pearson correlation coefficient between each index and water resources per capita, comprehensive production capacity of water supply, water consumption of urban residents, water consumption of industrial enterprises and the quantity of people affected by flood and drought, the correlation degree and significance test value can be obtained. The threshold value of the significance level test was set at 0.01 to carry out strict screening. Delete by the analysis of population density, population and 6 years old above college degree and above 6 years old,, water supply pipe network leakage rate loss modulus, water conservancy facilities as a result of small, regional GDP index correlation index, finally be related to urban water supply system resilience has 42 indicators (five kinds of index overlap, overlapping indicators calculated only once).

\section{Index selection is based on principal component factor analysis}

Since the two indexes of total water supply and total water use are highly correlated, it is not appropriate to conduct a factor analysis. After eliminating the total water use index after comprehensive consideration, factor analysis is conducted for the remaining 41 indexes. Before factor analysis of the data, $\mathrm{KMO}$ and Bartlett tests should be carried out, and the final results are shown in Table 1.

Sampling the Kaiser-Meyer-Olkin measure of adequacy

Bartlett's test for sphericity

\begin{tabular}{|c|c|}
\hline & 0.822 \\
\hline Approximate chi-square & 11443.479 \\
\hline$d f$ & 820 \\
\hline Sig. & 0.000 \\
\hline
\end{tabular}

Table 1. KMO and Bartlett test results

KMO value is 0.822 , and the corresponding Sig value is 0.000 , indicating that the original data used for the indicators in this paper are suitable for factor analysis. Factor analysis was performed on the data to get the total variance explained, as showed in Table 2 . The extraction method was the principal component analysis.

\begin{tabular}{|l|l|l}
\hline Composition & Initial eigenvalue & Sum of squares of the extraction load \\
\hline
\end{tabular}




\begin{tabular}{|c|c|c|c|c|c|c|c|c|c|}
\hline & Characteristic root & Variance $\%$ & Cumulative $\%$ & Characteristic root & Variance $\%$ & Cumulative \% & Characteristic root & Variance $\%$ & Cumulative \% \\
\hline 1 & 15.524 & 37.863 & 37.863 & 15.524 & 37.863 & 37.863 & 12.046 & 29.381 & 29.381 \\
\hline 2 & 5.888 & 14.361 & 52.224 & 5.888 & 14.361 & 52.224 & 5.712 & 13.933 & 43.314 \\
\hline 3 & 3.810 & 9.292 & 61.516 & 3.810 & 9.292 & 61.516 & 3.264 & 7.961 & 51.275 \\
\hline 4 & 1.905 & 4.646 & 66.163 & 1.905 & 4.646 & 66.163 & 2.796 & 6.820 & 58.095 \\
\hline 5 & 1.630 & 3.977 & 70.139 & 1.630 & 3.977 & 70.139 & 2.536 & 6.185 & 64.280 \\
\hline 6 & 1.512 & 3.689 & 73.828 & 1.512 & 3.689 & 73.828 & 2.352 & 5.736 & 70.016 \\
\hline 7 & 1.465 & 3.572 & 77.400 & 1.465 & 3.572 & 77.400 & 1.990 & 4.852 & 74.869 \\
\hline 8 & 1.136 & 2.770 & 80.170 & 1.136 & 2.770 & 80.170 & 1.756 & 4.282 & 79.151 \\
\hline 9 & 1.067 & 2.602 & 82.772 & 1.067 & 2.602 & 82.772 & 1.485 & 3.621 & 82.772 \\
\hline
\end{tabular}

Table 2. Total variance explained

According to the analysis in the above table, 9 common factors with feature roots greater than 1 were extracted through principal component analysis, and the single contribution rate of these 9 common factors changed slightly after the factor rotation, but the cumulative value of the contribution rate was $82.77 \%$, which did not change before and after the factor rotation. Combined with the factor load matrix, the correlation between these 9 common factors and specific indicators is further analyzed, so as to further classify and reduce the dimension of indicators. According to the impact load coefficient, the load coefficient is set to be above 0.5 . Finally, 14 indexes are deleted and 27 indexes are retained, this is shown in Table 3.

\begin{tabular}{|c|c|}
\hline Systems & Indicators \\
\hline \multirow{6}{*}{$\begin{array}{l}\text { Water source } \\
\qquad B_{1}\end{array}$} & Reservoir capacity at the year-end $C_{1}$ \\
\hline & Quantity of permanent residents at the year-end $C_{2}$ \\
\hline & Urbanization rate $C_{3}$ \\
\hline & Water resources per capita $C_{4}$ \\
\hline & Water consumption per $10,000 \mathrm{RMB}$ of industrial added value $C_{5}$ \\
\hline & Water consumption per $10,000 \mathrm{RMB}$ GDP $C_{6}$ \\
\hline \multirow{6}{*}{$\begin{array}{l}\text { Water plants } \\
\qquad B_{2}\end{array}$} & Domestic water consumption of urban residents $C_{7}$ \\
\hline & Comprehensive production capacity of water supply $C_{8}$ \\
\hline & $\begin{array}{l}\text { Personnel employed in urban units in the management of water conservancy, environment } \\
\text { and public facilities } C_{9}\end{array}$ \\
\hline & Urban sewage treatment rate $C_{10}$ \\
\hline & Total water supply $C_{11}$ \\
\hline & Investment in waste water treatment project has been completed $C_{12}$ \\
\hline \multirow{3}{*}{$\begin{array}{l}\text { Water supply and distribution } \\
\text { network } \\
B_{3}\end{array}$} & Length of water supply pipe $C_{13}$ \\
\hline & Density of water supply pipeline in built-up area $C_{14}$ \\
\hline & $\begin{array}{l}\text { Investment in fixed assets of water conservancy, environment and public facilities } \\
\text { management industry } C_{15}\end{array}$ \\
\hline \multirow{3}{*}{$\begin{array}{c}\text { Users } \\
B_{4}\end{array}$} & The quantity of people affected by floods and droughts $C_{16}$ \\
\hline & Percentage of urban basic medical insurance coverage at year-end $C_{17}$ \\
\hline & Quantity of people enrolled in unemployment insurance $C_{18}$ \\
\hline
\end{tabular}




\begin{tabular}{|l|l|}
\hline & Quantity of community health service centers $C_{19}$ \\
\hline State funds for education $C_{20}$ \\
\hline GDP per capita $C_{21}$ \\
\hline & Per capita disposable income of urban residents $C_{22}$ \\
\hline More old population dependency ratio $C_{23}$ \\
\hline Urban registered unemployment rate $C_{24}$ \\
\hline Natural population growth rate $C_{25}$ \\
\hline Economize water consumption $C_{26}$ \\
\hline Water consumption exceeding the planned quota $C_{27}$ \\
\hline
\end{tabular}

Table 3. Urban water supply system index classification

By referring to relevant toughness theoretical literature and combining with the above factor analysis results, the research team discussed together and regarded the above 9 common factors as the 9 factors affecting the toughness of the water supply and distribution system and classified and named them. Combined with the above toughness capability analysis framework, the following 5 index system dimensions were finally formed: Organization—Factor No. 1, Economy_Factor No. 2, Natural environment_Factor No. 3 and No. 7, Physics_—Factor No. 4, No. 8 and No. 9, Social—Factor No. 5 and No. 6. Factor classification results, this is shown in Table 4.

\begin{tabular}{|c|l|c|}
\hline Factor No. & \multicolumn{1}{|c|}{ Indicators } & Amount \\
\hline 1 & $C_{2}, C_{7}, C_{8}, C_{9}, C_{13}, C_{17}, C_{18}, C_{19}, C_{20}$ & 9 \\
\hline 2 & $C_{3}, C_{14}, C_{21}, C_{22}$ & 4 \\
\hline 3 & $C_{4}, C_{5}, C_{10}$ & 3 \\
\hline 4 & $C_{15}, C_{23}$ & 2 \\
\hline 5 & $C_{26}, C_{27}$ & 2 \\
\hline 6 & $C_{24}, C_{25}$ & 2 \\
\hline 7 & $C_{1}, C_{16}$ & 2 \\
\hline 8 & $C_{6}, C_{11}$ & 2 \\
\hline 9 & $C_{12}$ & 1 \\
\hline
\end{tabular}

Table 4. Factor classification results

\section{- Construction of toughness ability index system of urban water supply system}

Based on the above composition of urban water supply system, as showed in Figure 3, and combined with the result of factor analysis, construct an index system for the resilience of urban water supply systems, as showed in Table 5 .

\begin{tabular}{|l|l|l|c|l|}
\hline \multicolumn{1}{|c|}{ Dimension } & Water source & Water plants & Water supply and distribution network & \multicolumn{1}{c|}{ Users } \\
\hline Organization & $C_{2}$ & $C_{7}, C_{8}, C_{9}$ & $C_{13}$ & $\begin{array}{l}C_{17}, C_{18}, C_{19}, \\
C_{20}\end{array}$ \\
\hline Economy & $C_{3}$ & - & $C_{14}$ & $C_{21}, C_{22}$ \\
\hline Natural environment & $C_{1}, C_{4}, C_{5}$ & $C_{10}$ & - & $C_{16}$ \\
\hline Physics & $C_{6}$ & $C_{11}, C_{12}$ & $C_{15}$ & $C_{23}$ \\
\hline Social & - & - & - & $\begin{array}{l}C_{24}, C_{25}, C_{26}, \\
C_{27}\end{array}$ \\
\hline
\end{tabular}


Table 5. Index system for resilience capacity assessments

191

\section{Methods for assessing resilience of urban water supply systems}

\section{- Index weighting -- entropy weight method}

\section{Calculation steps}

(1) Standardization of index data

As the measurement units of each index are unified and the quantity is very different, the standardization of the index can eliminate the influence of different index dimensions on the program decision ${ }^{[21]}$. Indicators can be divided into two categories according to their nature. One is the bigger the better indicator, also known as the positive indicator. The other type is the smaller the better indicator, also known as the negative indicator. According to the characteristics of the indexes, the corresponding standardized treatment forms are adopted. Let's say I have $m$ samples, $n$ evaluation indicators, and $T$ years of data.

For the positive indicators:

$$
\bar{x}_{i j t}=\frac{x_{i j t}-\min _{1 \leq i \leq m} x_{i j t}}{\max _{1 \leq i \leq m} x_{i j t}-\min _{1 \leq i \leq m} x_{i j t}}
$$

For the negative indicators:

$$
\bar{x}_{i j t}=\frac{\max _{1 \leq i \leq m} x_{i j t}-x_{i j t}}{\max _{1 \leq i \leq m} x_{i j t}-\min _{1 \leq i \leq m} x_{i j t}}
$$

Where: $x_{i j t}$ is the original index value of index $j$ in city $i$ in the $t$ year, and $\bar{x}_{i j t}$ is the standardized value of index $j$ in city $i$ in the $t$ year. $i=1,2, \ldots, \mathrm{m} ; \mathrm{j}=1,2, \ldots, \mathrm{n} ; \mathrm{t}=1,2, \ldots, \mathrm{T}$.

(2) Calculate the characteristic proportion of the appraisal value in the tyear of city $i$ of index $j$ :

$$
P_{i j t}=\frac{\bar{x}_{i j t}}{\sum_{i=1}^{m} \bar{x}_{i j t}}, i=1,2, \ldots, \mathrm{m} ; j=1,2, \ldots, \mathrm{n}
$$

(3) Calculate the information entropy $\left(E_{j t}\right)$ and differential coefficient $\left(d_{j t}\right)$ of the $j$ index in the $t$ year:

$$
\begin{aligned}
& E_{j t}=-\frac{1}{\ln (n)} \sum_{i=1}^{m} P_{i j t} \ln \left(P_{i j t}\right), i=1,2, \ldots, \mathrm{m} ; \mathrm{j}=1,2, \ldots, \mathrm{n} \\
& d_{i j t}=1-E_{j t}, j=1,2, \ldots, \mathrm{n}
\end{aligned}
$$

(4) Calculate the weight of each evaluation index in the $t$ year: 


$$
W_{j t}=\frac{d_{j t}}{\sum_{j=1}^{n} d_{j t}}
$$

(5) In order to obtain the uniform weight of each index, the average value of the sample index data in $T$ year is taken, and using arithmetic average method to calculate the uniform weight of the index:

$$
\bar{W}_{j t}=\sum_{t=1}^{T} W_{j t}
$$

\section{Calculation of unified weight}

When determining the weight of the comprehensive evaluation index of toughness capacity of urban water supply system with multi-year and multi-region direction, the weight of the index calculated varies from year to year due to the different data each year. Therefore, the comprehensive evaluation value lacks comparability in the longitudinal comparison, which affects the final evaluation result ${ }^{[22]}$. Therefore, this article put forward considering the index data of different areas more than one year averaged them, based on this data to get the weight of a unified, and the weight as a fixed value, when to stay also adopt the unified when the appraisal object weight to calculate the toughness value, so quickly to evaluate an area water supply and distribution system resilience, And make the calculated results more comparable.

Therefore, when calculating the unified weight, this paper adopts the original data of toughness index of the water supply and distribution system from 31 provinces and cities in China from 2014 to 2019. The results of average weight calculation by MATLAB 7.0 software are shown in Table 6 .

\begin{tabular}{|c|c|c|c|c|c|c|c|c|}
\hline Systems & Indicators & 2019 & 2018 & 2017 & 2016 & 2015 & 2014 & Average weight \\
\hline \multirow{4}{*}{$\begin{array}{c}B_{1} \\
0.3300\end{array}$} & $C_{1}$ & 0.072 & 0.072 & 0.075 & 0.075 & 0.067 & 0.063 & 0.071 \\
\cline { 2 - 8 } & $C_{2}$ & 0.013 & 0.013 & 0.014 & 0.014 & 0.015 & 0.015 & 0.014 \\
\cline { 2 - 8 } & $C_{3}$ & 0.011 & 0.011 & 0.012 & 0.012 & 0.013 & 0.014 & 0.012 \\
\cline { 2 - 8 } & $C_{4}$ & 0.217 & 0.205 & 0.214 & 0.230 & 0.234 & 0.235 & 0.223 \\
\cline { 2 - 8 } & $C_{5}$ & 0.006 & 0.006 & 0.006 & 0.005 & 0.005 & 0.006 & 0.006 \\
\hline \multirow{4}{*}{$\begin{array}{c}B_{2} \\
0.1689\end{array}$} & $C_{6}$ & 0.005 & 0.004 & 0.005 & 0.005 & 0.005 & 0.005 & 0.005 \\
\cline { 2 - 8 } & $C_{7}$ & 0.005 & 0.005 & 0.005 & 0.005 & 0.005 & 0.006 & 0.005 \\
\cline { 2 - 8 } & $C_{8}$ & 0.043 & 0.045 & 0.045 & 0.046 & 0.044 & 0.046 & 0.045 \\
\cline { 2 - 8 } & $C_{9}$ & 0.016 & 0.016 & 0.018 & 0.018 & 0.018 & 0.020 & 0.018 \\
\cline { 2 - 8 } & $C_{10}$ & 0.006 & 0.008 & 0.004 & 0.005 & 0.004 & 0.005 & 0.005 \\
\hline \multirow{4}{*}{$B_{3}$} & $C_{12}$ & 0.040 & 0.040 & 0.042 & 0.042 & 0.042 & 0.043 & 0.041 \\
\cline { 2 - 8 } 0.1098 & $C_{13}$ & 0.046 & 0.045 & 0.049 & 0.050 & 0.052 & 0.051 & 0.055 \\
\hline
\end{tabular}




\begin{tabular}{|c|c|c|c|c|c|c|c|c|}
\hline & $C_{15}$ & 0.035 & 0.029 & 0.027 & 0.025 & 0.026 & 0.026 & 0.028 \\
\hline \multirow{7}{*}{} & $C_{16}$ & 0.005 & 0.006 & 0.017 & 0.011 & 0.007 & 0.006 & 0.009 \\
\cline { 2 - 8 } & $C_{17}$ & 0.036 & 0.046 & 0.048 & 0.041 & 0.039 & 0.038 & 0.041 \\
\cline { 2 - 8 } & $C_{18}$ & 0.044 & 0.043 & 0.045 & 0.045 & 0.045 & 0.040 & 0.044 \\
\cline { 2 - 8 } & $C_{19}$ & 0.028 & 0.028 & 0.030 & 0.031 & 0.033 & 0.034 & 0.030 \\
\cline { 2 - 8 } 0.3912 & $C_{20}$ & 0.029 & 0.028 & 0.028 & 0.029 & 0.027 & 0.027 & 0.028 \\
\cline { 2 - 8 } & $C_{21}$ & 0.034 & 0.037 & 0.039 & 0.045 & 0.041 & 0.039 & 0.039 \\
\cline { 2 - 8 } & $C_{22}$ & 0.058 & 0.062 & 0.064 & 0.065 & 0.064 & 0.049 & 0.060 \\
\cline { 2 - 8 } & $C_{23}$ & 0.020 & 0.017 & 0.017 & 0.013 & 0.013 & 0.012 & 0.015 \\
\cline { 2 - 8 } & $C_{24}$ & 0.022 & 0.030 & 0.017 & 0.017 & 0.022 & 0.033 & 0.024 \\
\cline { 2 - 8 } & $C_{25}$ & 0.020 & 0.020 & 0.017 & 0.014 & 0.016 & 0.017 & 0.017 \\
\cline { 2 - 8 } & $C_{26}$ & 0.076 & 0.090 & 0.066 & 0.069 & 0.070 & 0.068 & 0.073 \\
\hline
\end{tabular}

Table 6. Weight of indexes for resilience capacity assessment

\section{- A cloud model for assessing resilience of urban water supply systems}

\section{Cloud model concept}

Academician Li Deyi ${ }^{[23]}$ first defined the concept of a cloud model, which is a mathematical model in which a qualitative description and quantitative concept transform each other with uncertainty. The existing qualitative evaluation mainly has the problems of subjectivity and arbitrariness. The advantage of the cloud model is that it can overcome the above disadvantages and effectively evaluate the evaluation object.

Let $u$ be a quantitative domain expressed by numerical value, and $C$ be a qualitative concept on $U$. If the quantitative value $x \in U$ is a random realization of the qualitative concept $C$, the certainty of $x$ for $C, u(x) \in[0,1]$ is a random number with a stable tendency:

$$
u: U \rightarrow[0,1], \forall x \in U, x \in u(x)
$$

Then the distribution of $x$ in the domain $U$ is called the cloud model (cloud for short), decided as $C(x)$; Each $x$ is called a cloud droplet.

When representing a concept as a whole, three digital features are used to realize it, namely, expectation Ex, entropy En and hypermetropy He, as showed in Figure 5. 


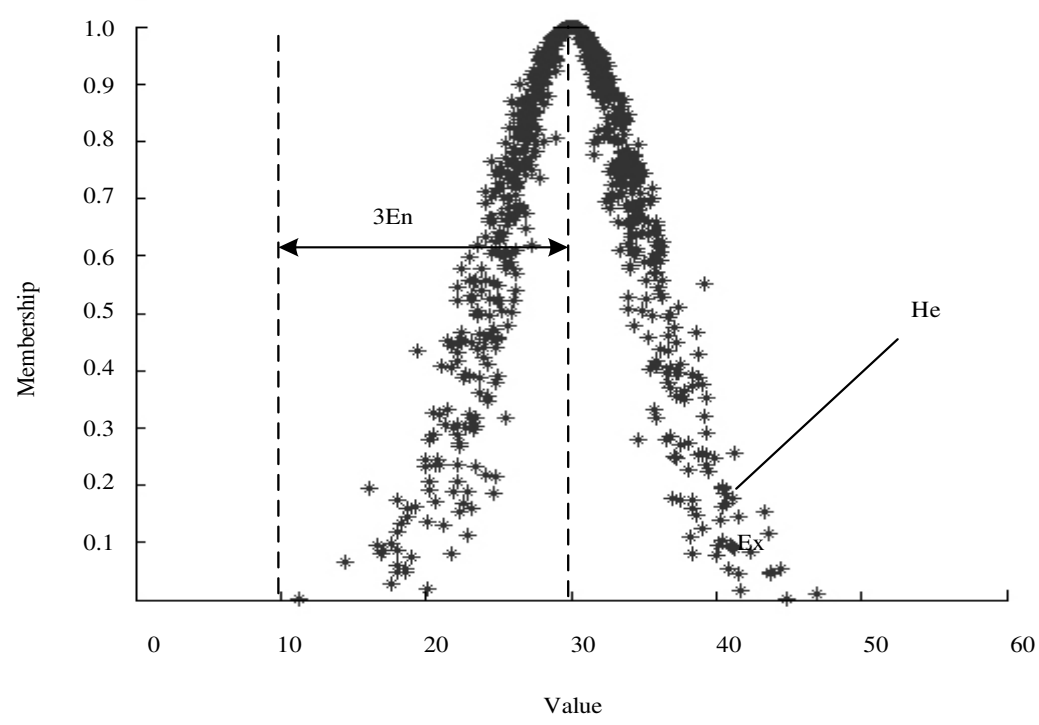

Figure 5. Schematic illustration of the digital features of the cloud

\section{Cloud model construction steps}

When determining the membership degree, the traditional fuzzy membership degree is a fixed value. However, when the cloud model is used to calculate the membership degree of the index in the cloud, the membership degree of the index for the evaluation set is not accurate and unique, thus reducing the subjectivity and difficult ${ }^{[24]}$.

Establishment steps of multi-level comprehensive evaluation model based on cloud model ${ }^{[25-26]}$ :

(1) Factor field $U$ and comment field $V$ are established for the evaluation objects.

(2) The index weight $W$ calculated in Section 2.3 is adopted.

(3) A single factor evaluation was conducted between $U$ and $V$, and a fuzzy relational matrix $R$ was established. Let the 253 factor $i(i=1,2, \ldots, n)$ corresponding grade $j(j=1,2, \ldots, m)$ the upper boundary value is $x_{i j}$ and the lower boundary value is $x_{i j}^{\prime}$, then the qualitative concept of level $\mathrm{j}$ corresponding to factor I can be represented by the normal cloud model, where:

$$
E x_{i j}=\left(x_{i j}+x_{i j}\right) / 2
$$

Since the boundary value is the transition value of two adjacent levels, and the membership degree of the two levels is equal, there are:

$$
\exp \left\{-\frac{\left(x_{i j}-x_{i j}\right)^{2}}{8\left(E n_{i j}\right)^{2}}\right\}=0.5
$$

Namely

$$
E n_{i j}=\left(x_{i j}-x_{i j}\right) / 2.355
$$

(4) The cloud model membership matrix $C^{\prime}=\left(c_{i j}\right)_{n \times m}$ for each index corresponding to each level of the system layer is calculated from the index value of the evaluation object, where $C_{i j}$ is the average value under different membership degrees 
(normal cloud generator under $X$ condition is run $N$ times) :

$$
C_{i j}=\frac{1}{N} \sum_{k=1}^{n} C_{i j}^{k}
$$

(5) The fuzzy subset $B^{\prime}$ on the evaluation set $V$ of the system layer is obtained through the fuzzy transformation between the weight set $W^{\prime}$ of the indicator layer and the membership matrix $C^{\prime}$ :

$$
B^{\prime}=W^{\prime *} C^{\prime}=\left(b_{1}, b_{2}, \cdots, b_{m}\right)
$$

In the formula:

$$
b_{j}=\sum_{i=1}^{n} w_{i} c_{i j}, j=1, \cdots, \quad m
$$

$b_{j}$ represents the membership degree of the object to be evaluated to the comments in Article $j$. According to the principle of maximum membership degree, the $i$ evaluation grade corresponding to the maximum membership degree of article $j$ comments was selected as the result of system-level evaluation.

(6) Similarly, the fuzzy subset $B$ of the target layer is obtained by the high-level fuzzy transformation between the weight set $W$ of the system layer and the fuzzy subset $B^{\prime}$ of the system layer. Finally, according to the principle of maximum degree of membership, the comprehensive evaluation grade of the target layer is obtained.

\section{Case analysis}

\section{- Study areas and data source}

Qingdao has more people and less water, and the spatial and temporal distribution of precipitation is uneven. Especially in recent years, severe water supply crisis caused by extreme weather has appeared. With the rapid economic growth and the continuous improvement of urbanization, the safety of the water supply has become an important factor restricting the sustainable economic and social development of Qingdao. Therefore, Qingdao has built a large water supply and distribution system of "three sources" raw water supply, "four vertical and three horizontal" pipe network transmission and distribution in the main urban area, and "one ring and three lines" unified allocation in Qingdao. This paper takes Qingdao as an example. The data are from 2010-2019 "Qingdao Water Resources Bulletin", "Qingdao Statistical Yearbook", "Qingdao Statistical Bulletin", "Shandong Province Statistical Yearbook" and so on. At present, the distribution of water resources in Qingdao is shown in Figure 6. The status quo of the main water supply projects in Qingdao is shown in Table 7. 


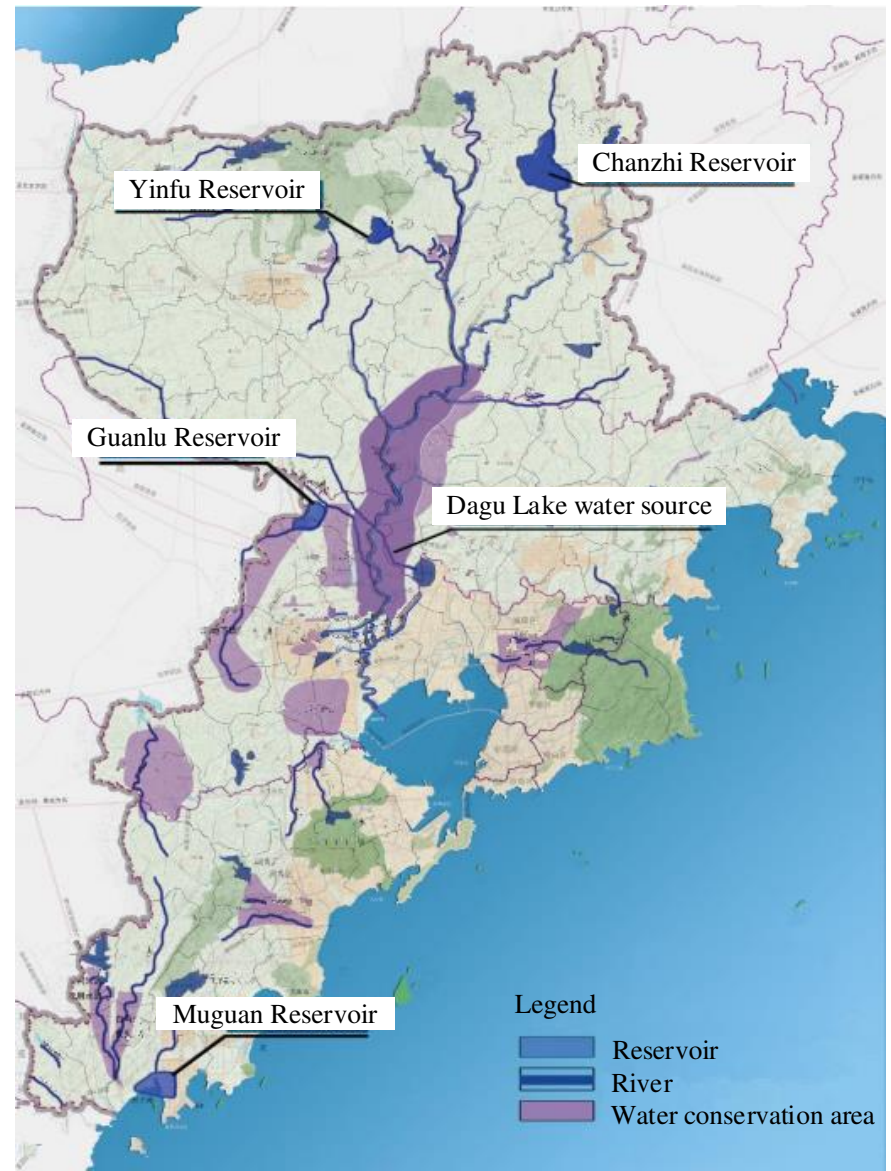

Figure 6. Distribution of water resources in Qingdao(The figure shows the distribution of reservoirs, rivers and water conservation areas in Qingdao)

\begin{tabular}{|c|l|l|l|c|}
\hline No. & \multicolumn{1}{|c|}{ Water sources } & \multicolumn{1}{|c|}{ Water plants } & \multicolumn{1}{|c|}{ Water supply area } & $\begin{array}{c}\text { Water supply capacity* } \\
\left(10000 \mathrm{~m}^{3} / \mathrm{d}\right)\end{array}$ \\
\hline 1 & Chanzhi reservoir & Zhangezhuang waterworks & Laixi city & 10 \\
\hline 2 & Chanzhi reservoir & Baishahe waterworks & Five districts in Qingdao & 8 \\
\hline 3 & Yinfu reservoir & Xingping waterworks & Pingdu city & 2 \\
\hline 4 & Yinfu reservoir & Baishahe waterworks & Five districts in Qingdao & 4 \\
\hline 5 & Huangshan reservoir & Xingping waterworks & Pingdu city & 0.3 \\
\hline 6 & Zhangling water source & Yunshan waterworks & Pingdu city & 2.2 \\
\hline 7 & Wangquan reservoir & Shibei waterworks & Jimo city & 0.2 \\
\hline 8 & Songhuaquan reservoir & Shibei waterworks & Jimo city & 12 \\
\hline 9 & Nuocheng reservoir & Tongji waterworks & Jimo city & 2 \\
\hline 10 & Shipeng reservoir & Shinan waterworks & Jimo city & 1 \\
\hline 11 & Moshuihe water source & Wuqi waterworks & Jimo city & 10 \\
\hline 12 & Water source & Baishahe waterworks & Five districts in Qingdao & 0.5 \\
\hline 13 & Qingnian reservoir & Zhuanglitou waterworks & Jiaozhou city & 2 \\
\hline
\end{tabular}




\begin{tabular}{|c|l|l|l|c|}
\hline 14 & Shanzhou reservoir & Zhuanglitou waterworks & Jiaozhou city & 1 \\
\hline 15 & Shuyuan reservoir & Jiangjiazhuang waterworks & Five districts in Qingdao & 1.5 \\
\hline 16 & Laoshan reservoir & Laoshan waterworks & Five districts in Qingdao & 7.5 \\
\hline 17 & Baishahe water source & $\begin{array}{l}\text { Xiazhuang waterworks } \\
\text { Shuanglong waterworks }\end{array}$ & Five districts in Qingdao & 3.5 \\
\hline 18 & Xiaozhushan reservoir & Xiaozhushan waterworks & Huangdao district & 2 \\
\hline 19 & Jilihe reservoir & Gaojiatai waterworks & Huangdao district & 5.5 \\
\hline 20 & Tieshan reservoir & No.3 waterworks & Huangdao district & 2 \\
\hline 21 & Douyazi reservoir & No.5 waterworks & Huangdao district & 5.8 \\
\hline 22 & Fenghe water source & No.2 and No.4 waterworks & Huangdao district & 23.5 \\
\hline 23 & Jihongtan reservoir & Xianjiazhai waterworks & Five districts in Qingdao & 1.5 \\
\hline 24 & Jihongtan reservoir & Kaifaqu waterworks & Jiaozhou city & 10 \\
\hline 25 & Jihongtan reservoir & Guanjialou waterworks & Huangdao district & 16 \\
\hline 26 & Jihongtan reservoir & Hongshiya waterworks & Huangdao district & 3 \\
\hline 27 & Jihongtan reservoir & Western water supply office & Five districts in Qingdao & 10 \\
\hline 28 & Chanzhi reservoir & Huashan waterworks & $\begin{array}{l}\text { Jimo city } \\
\text { Chengyang district } \\
\text { Laoshan district }\end{array}$ & \\
\hline
\end{tabular}

Table 7. Present situations of the main water source and water supply project in Qingdao(Data from Qingdao Water Resources Construction and Allocation "Thirteenth Five-Year Plan")

According to the established toughness capability index system and index standard, the normal cloud model is used to represent the grade standard of each index in Equations (9) and (11).

Indicators with higher weights were successively selected, such as per capita water resource $C_{4}$, completed investment of wastewater treatment project $C_{12}$, length of water supply pipeline $C_{13}$, and water consumption saving $C_{26}$. Equation (1) and cloud matrix R were used to establish the normal cloud membership function of evaluation index standard, as showed in Figure 7. The numbers 1, 2, 3, 4 and 5 on the ordinate of Figure 7 correspond to the low level, a slightly lower level, medium level, slightly higher level and high level of the evaluation grade respectively. The $X$ conditional cloud generator was used to obtain the membership matrix of each index value. Assuming that the number of cloud droplets generated was $N=800$, the data of Qingdao City from 2010 to 2019 were substituted into the repeated calculation for 800 times to obtain the average membership of each evaluation grade. 


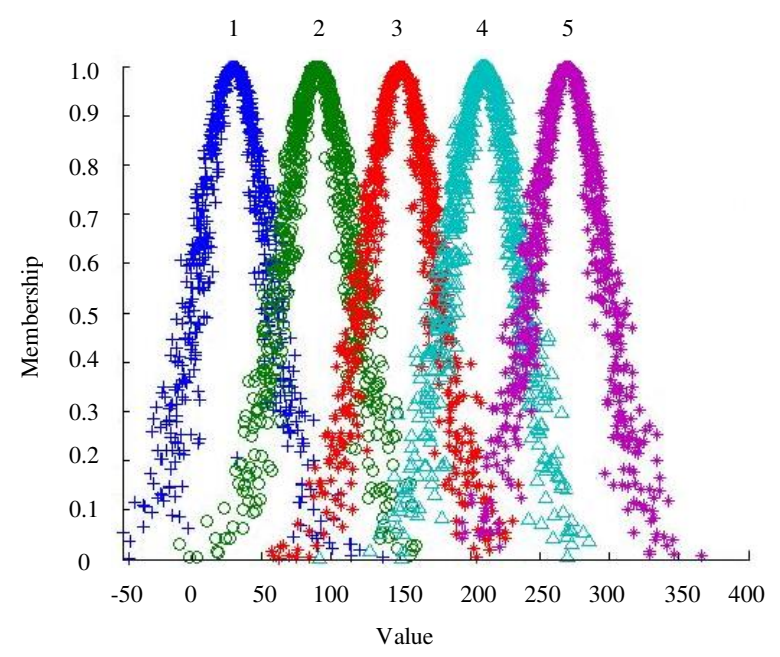

(a)

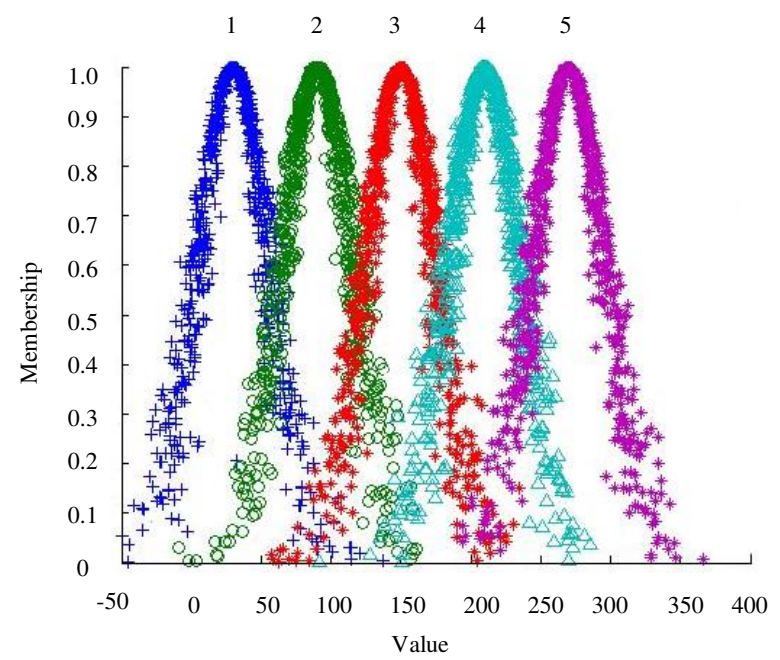

(c)

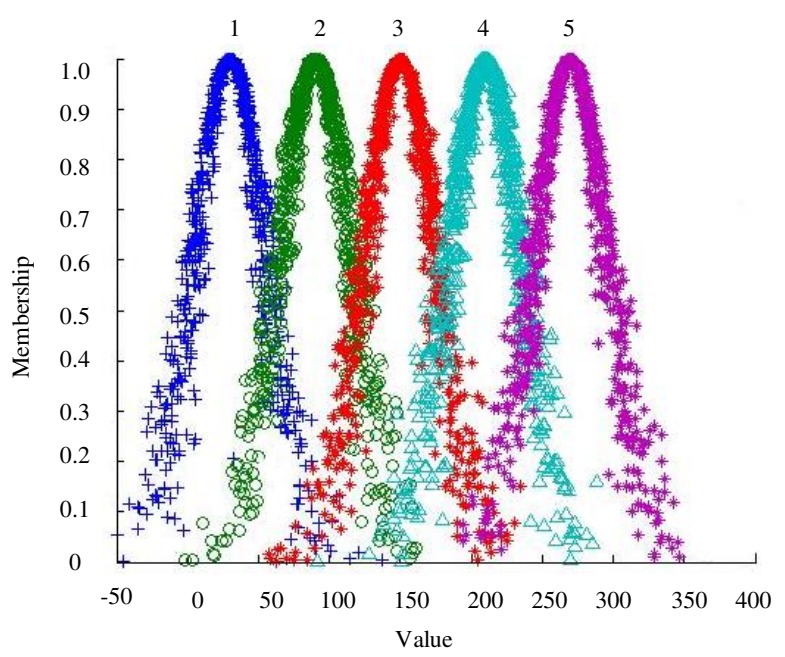

(b)

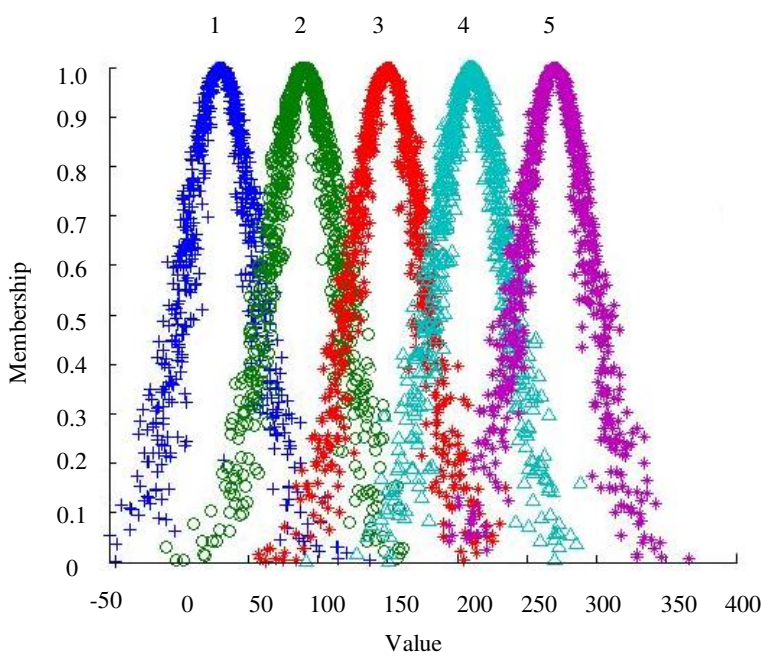

(d)

Figure 7. Membership functions for Normal cloud(（a）Take the index of water resources per capita as an example,

(b) Take waste water treatment project investment index as an example, (c) Take the length index of water supply pipeline as an example, (d) Take the index of saving water consumption as an example)

Finally, according to steps (5) and (6) of the cloud model, the comprehensive evaluation results are obtained, as showed in Table 8 and Figure 8 . In order to ensure the integrity of the evaluation, the whole water supply system $\left(B_{0}\right)$ is added as one of the toughness evaluation indexes.

\begin{tabular}{|c|c|c|c|c|c|c|c|}
\hline \multirow{2}{*}{ Year } & Systems & Low level & $\begin{array}{c}\text { Slightly lower } \\
\text { level }\end{array}$ & Medium level & $\begin{array}{c}\text { Slightly higher } \\
\text { level }\end{array}$ & High level & Rating \\
\hline \multirow{7}{*}{2019} & $B_{0}$ & 0.069 & 0.063 & 0.087 & 0.079 & 0.032 & 3 \\
\cline { 2 - 8 } & $B_{1}$ & 0.194 & 0.035 & 0.080 & 0.013 & 0.008 & 1 \\
\cline { 2 - 8 } & $B_{2}$ & 0.007 & 0.062 & 0.040 & 0.061 & 0.012 & 2 \\
\cline { 2 - 8 } & $B_{3}$ & 0.000 & 0.015 & 0.040 & 0.056 & 0.001 & 4 \\
\cline { 2 - 8 } & $B_{4}$ & 0.011 & 0.101 & 0.128 & 0.148 & 0.068 & 4 \\
\hline 2018 & $B_{0}$ & 0.077 & 0.049 & 0.087 & 0.073 & 0.024 & 3 \\
\hline
\end{tabular}




\begin{tabular}{|c|c|c|c|c|c|c|c|}
\hline & $B_{1}$ & 0.224 & 0.005 & 0.043 & 0.016 & 0.005 & 1 \\
\hline & $B_{2}$ & 0.007 & 0.076 & 0.050 & 0.042 & 0.008 & 2 \\
\hline & $B_{3}$ & 0.000 & 0.029 & 0.049 & 0.029 & 0.000 & 3 \\
\hline & $B_{4}$ & 0.006 & 0.081 & 0.150 & 0.148 & 0.053 & 3 \\
\hline \multirow{5}{*}{2017} & $B_{0}$ & 0.079 & 0.056 & 0.101 & 0.052 & 0.022 & 3 \\
\hline & $B_{1}$ & 0.229 & 0.007 & 0.040 & 0.014 & 0.007 & 1 \\
\hline & $B_{2}$ & 0.008 & 0.074 & 0.070 & 0.015 & 0.004 & 2 \\
\hline & $B_{3}$ & 0.000 & 0.034 & 0.066 & 0.030 & 0.000 & 3 \\
\hline & $B_{4}$ & 0.006 & 0.095 & 0.175 & 0.105 & 0.050 & 3 \\
\hline \multirow{5}{*}{2016} & $B_{0}$ & 0.068 & 0.086 & 0.144 & 0.050 & 0.007 & 3 \\
\hline & $B_{1}$ & 0.197 & 0.064 & 0.130 & 0.016 & 0.005 & 1 \\
\hline & $B_{2}$ & 0.007 & 0.081 & 0.024 & 0.063 & 0.014 & 2 \\
\hline & $B_{3}$ & 0.001 & 0.028 & 0.051 & 0.031 & 0.000 & 3 \\
\hline & $B_{4}$ & 0.006 & 0.123 & 0.234 & 0.077 & 0.007 & 3 \\
\hline \multirow{5}{*}{2015} & $B_{0}$ & 0.071 & 0.065 & 0.125 & 0.055 & 0.009 & 3 \\
\hline & $B_{1}$ & 0.202 & 0.005 & 0.046 & 0.085 & 0.014 & 1 \\
\hline & $B_{2}$ & 0.011 & 0.085 & 0.069 & 0.006 & 0.002 & 2 \\
\hline & $B_{3}$ & 0.000 & 0.025 & 0.075 & 0.011 & 0.000 & 3 \\
\hline & $B_{4}$ & 0.007 & 0.118 & 0.230 & 0.063 & 0.011 & 3 \\
\hline \multirow{5}{*}{2014} & $B_{0}$ & 0.070 & 0.073 & 0.122 & 0.031 & 0.023 & 3 \\
\hline & $B_{1}$ & 0.197 & 0.004 & 0.039 & 0.042 & 0.052 & 1 \\
\hline & $B_{2}$ & 0.010 & 0.085 & 0.055 & 0.007 & 0.001 & 2 \\
\hline & $B_{3}$ & 0.002 & 0.030 & 0.082 & 0.008 & 0.000 & 3 \\
\hline & $B_{4}$ & 0.009 & 0.138 & 0.231 & 0.037 & 0.014 & 3 \\
\hline \multirow{5}{*}{2013} & $B_{0}$ & 0.084 & 0.090 & 0.108 & 0.018 & 0.032 & 3 \\
\hline & $B_{1}$ & 0.222 & 0.013 & 0.044 & 0.027 & 0.067 & 1 \\
\hline & $B_{2}$ & 0.014 & 0.095 & 0.047 & 0.003 & 0.005 & 2 \\
\hline & $B_{3}$ & 0.006 & 0.025 & 0.076 & 0.012 & 0.000 & 3 \\
\hline & $B_{4}$ & 0.020 & 0.171 & 0.196 & 0.018 & 0.023 & 3 \\
\hline \multirow{5}{*}{2012} & $B_{0}$ & 0.084 & 0.078 & 0.082 & 0.015 & 0.035 & 1 \\
\hline & $B_{1}$ & 0.177 & 0.003 & 0.020 & 0.028 & 0.077 & 1 \\
\hline & $B_{2}$ & 0.013 & 0.096 & 0.050 & 0.006 & 0.000 & 2 \\
\hline & $B_{3}$ & 0.002 & 0.032 & 0.079 & 0.010 & 0.000 & 3 \\
\hline & $B_{4}$ & 0.058 & 0.145 & 0.148 & 0.008 & 0.023 & 3 \\
\hline \multirow{5}{*}{2011} & $B_{0}$ & 0.099 & 0.076 & 0.071 & 0.035 & 0.013 & 1 \\
\hline & $B_{1}$ & 0.179 & 0.003 & 0.040 & 0.081 & 0.021 & 1 \\
\hline & $B_{2}$ & 0.015 & 0.121 & 0.046 & 0.003 & 0.000 & 2 \\
\hline & $B_{3}$ & 0.002 & 0.033 & 0.077 & 0.009 & 0.000 & 3 \\
\hline & $B_{4}$ & 0.096 & 0.130 & 0.107 & 0.018 & 0.016 & 2 \\
\hline \multirow{3}{*}{2010} & $B_{0}$ & 0.108 & 0.082 & 0.081 & 0.016 & 0.018 & 1 \\
\hline & $B_{1}$ & 0.213 & 0.028 & 0.099 & 0.034 & 0.011 & 1 \\
\hline & $B_{2}$ & 0.016 & 0.073 & 0.018 & 0.002 & 0.053 & 2 \\
\hline
\end{tabular}




\begin{tabular}{|l|l|l|l|l|l|l|l|}
\hline & $B_{3}$ & 0.000 & 0.018 & 0.072 & 0.008 & 0.000 & 3 \\
\cline { 2 - 7 } & $B_{4}$ & 0.090 & 0.149 & 0.095 & 0.010 & 0.012 & 2 \\
\hline
\end{tabular}

Table 8. Comprehensive assessment results of resilience capacity
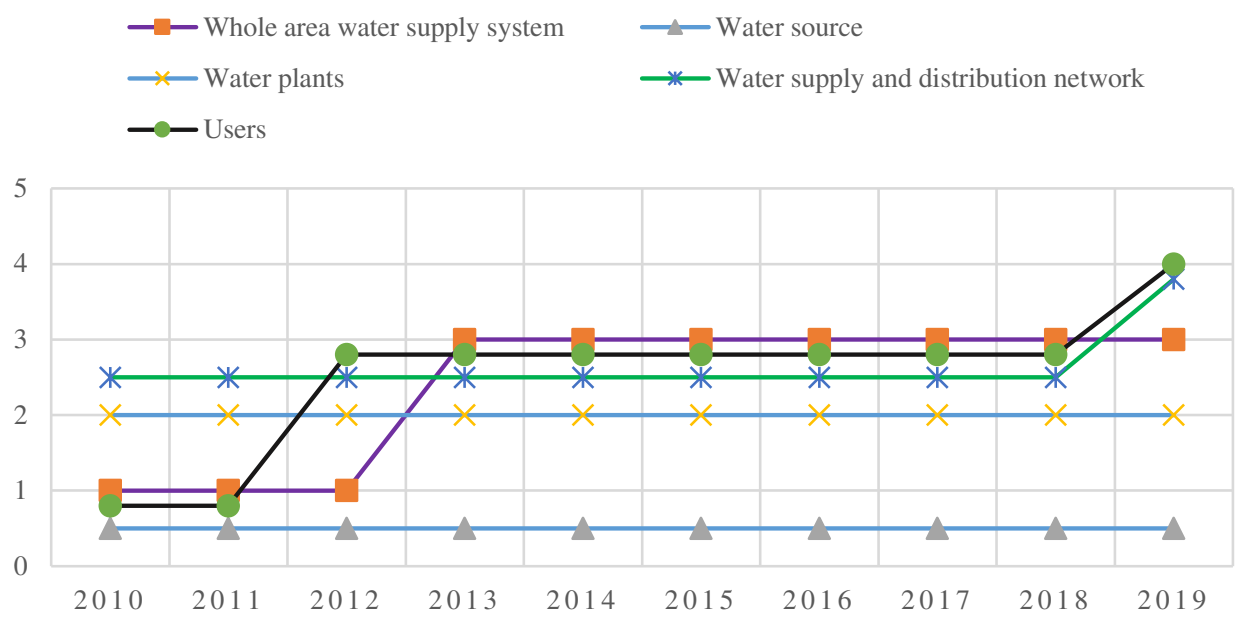

Figure 8. Trends in cloud assessment levels of resilience capacity

Based on the comprehensive evaluation results, it can be seen that from 2013 to 2019, the toughness level of Qingdao's

water supply and distribution system was at a medium level, and in 2010, 2011 and 2012, it was at a low level. The overall development trend of the toughness level was good. Among all water supply subsystems, the resilience of water source system is always at a low level and has not been improved in the past ten years. The toughness of user system was at a low level in 2010 and 2011. At a medium level from 2012 to 2018, and at a high level in 2019, showing a step-like upward trend. The toughness of the water plant system has been at a low level for ten years. The toughness of the water supply and distribution pipe network system has been stable and at a medium level from 2010 to 2018, and has risen to a higher level in 2019. Since 2012, toughness of all subsystems has been ranked in order. The user system and the water supply and distribution pipe network system have the same toughness and rank the highest, followed by the water plant system and the water source system.

- Calculation of Resilience Capacity of Index Comprehensive Evaluation Index Method Based on Entropy Weight

The weight of the regional water supply and distribution system adopts the unified weight value $W_{j}$ calculated by the Entropy Weight, and the toughness evaluation index system of the regional urban water supply system constructed in Section 3.2. The evaluation indexes go through the process of data acquisition, input and data standardization.

Here the original index data is standardized by the extremum method (the Min-max normalization method) to eliminate the dimensionality effect.

The normalized index $r_{i j}$ of $j$ evaluation indexes in the $i$ year was calculated, and then the comprehensive evaluation index $V$ $=W_{j} * r_{i j}$ was synthesized.In the case of 2019 , the calculation process is similar for the other years. The final toughness 
capacity calculation results are shown in Table 9 and Figure 9.

\begin{tabular}{|c|c|c|c|c|}
\hline Systems & Index & Standardized values & Index weight & Composite \\
\hline \multirow{6}{*}{$\begin{array}{c}B_{1} \\
0.3300\end{array}$} & $C_{1}$ & 0.4013 & 0.0706 & 0.0283 \\
\hline & $C_{2}$ & 0.0000 & 0.0140 & 0.0000 \\
\hline & $C_{3}$ & 1.0000 & 0.0121 & 0.0121 \\
\hline & $C_{4}$ & 0.2621 & 0.2226 & 0.0583 \\
\hline & $C_{5}$ & 0.9464 & 0.0057 & 0.0054 \\
\hline & $C_{6}$ & 1.0000 & 0.0049 & 0.0049 \\
\hline \multirow{6}{*}{$\begin{array}{c}B_{2} \\
0.1689\end{array}$} & $C_{7}$ & 0.0000 & 0.0051 & 0.0000 \\
\hline & $C_{8}$ & 1.0000 & 0.0448 & 0.0448 \\
\hline & $C_{9}$ & 0.9231 & 0.0175 & 0.0162 \\
\hline & $C_{10}$ & 1.0000 & 0.0053 & 0.0053 \\
\hline & $C_{11}$ & 0.9529 & 0.0414 & 0.0395 \\
\hline & $C_{12}$ & 0.4000 & 0.0548 & 0.0219 \\
\hline \multirow{3}{*}{$\begin{array}{c}B_{3} \\
0.1098\end{array}$} & $C_{13}$ & 1.0000 & 0.0487 & 0.0487 \\
\hline & $C_{14}$ & 0.0000 & 0.0331 & 0.0000 \\
\hline & $C_{15}$ & 1.0000 & 0.0280 & 0.0280 \\
\hline \multirow{12}{*}{$\begin{array}{c}B_{4} \\
0.3912\end{array}$} & $C_{16}$ & 0.0000 & 0.0086 & 0.0000 \\
\hline & $C_{17}$ & 1.0000 & 0.0414 & 0.0414 \\
\hline & $C_{18}$ & 1.0000 & 0.0439 & 0.0439 \\
\hline & $C_{19}$ & 1.0000 & 0.0304 & 0.0304 \\
\hline & $C_{20}$ & 1.0000 & 0.0279 & 0.0279 \\
\hline & $C_{21}$ & 1.0000 & 0.0393 & 0.0393 \\
\hline & $C_{22}$ & 1.0000 & 0.0602 & 0.0602 \\
\hline & $C_{23}$ & 0.0000 & 0.0154 & 0.0000 \\
\hline & $C_{24}$ & 0.1944 & 0.0236 & 0.0046 \\
\hline & $C_{25}$ & 0.2765 & 0.0174 & 0.0048 \\
\hline & $C_{26}$ & 0.6667 & 0.0734 & 0.0489 \\
\hline & $C_{27}$ & 0.9080 & 0.0098 & 0.0089 \\
\hline
\end{tabular}

Table 9. Toughness capability evaluation indexes are comprehensive evaluation index 


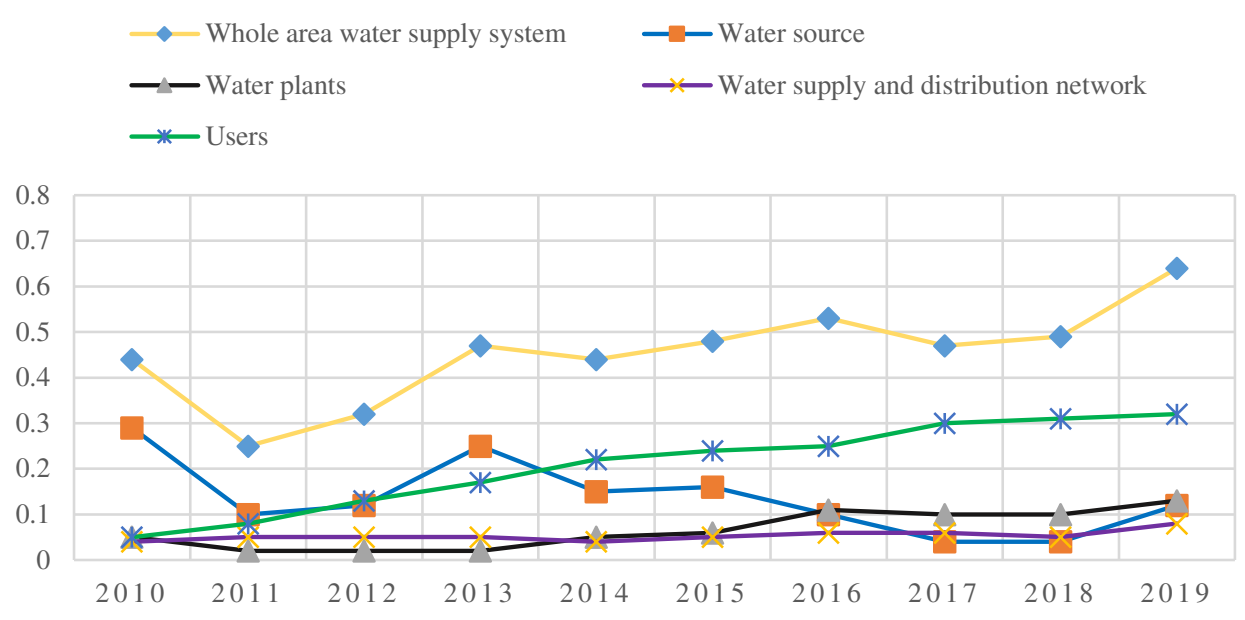

Figure 9. Comprehensive evaluation index trend of toughness capability by entropy weight method

It can be seen from Figure 9 that, in general, toughness of Qingdao's water supply and distribution system during the period from 2010 to 2019 showed a wavy upward trend, although some years showed a decline.Each subsystem toughness index change trend of water supply, water supply subsystem comprehensive index of the whole year of a downward trend and the final year of the initial drop apparently, user subsystem comprehensive index is on the rise and growth of apparent, waterworks subsystem have been falling and rising trend, for water distribution network system is slightly rising trend, the growth is not obvious.

\section{- Comparison of the two evaluation results}

By comparing the multi-level comprehensive evaluation results of the cloud model with those of the comprehensive evaluation index method of entropy weight index, it can be seen that the latter is to calculate the fixed comprehensive index value, the index value and index weight into positive correlation, while the reflect of the subsystems of the water supply and distribution system and the overall dynamic change trend, However, it is difficult to describe in detail the degree to which each evaluation unit belongs to a certain level.For example, in the example, the index per capita water resource has a large weight. If the index comprehensive evaluation index method based on Entropy Weight is adopted to calculate, it will have a large and positive impact on the toughness of the system. If the multi-level evaluation model of the cloud model is used, the toughness level is at a low level, which has a great but negative impact on the toughness of the system.Moreover, the multi-level evaluation result based on cloud model is more flexible, which examines the extent to which the evaluation unit belongs to a certain level, and the boundary of the level also changes within a certain acceptable range. All subsystems in the water supply and distribution system determine the toughness of a water supply and distribution system. However, the overall toughness of the water supply and distribution system is not simply composed of the superposition of all subsystems, but the interaction and mutual influence of all subsystems. Therefore, toughness capability is more in line with the fuzzy connotation of the cloud model theory.

\section{- Discussion}


According to the further analysis of the status quo of water supply in Qingdao, the economic development level and urbanization rate of Qingdao have been improving year by year in recent years, and the social security system has been increasingly improved. In 2019, the per capita GDP of Qingdao has exceeded 119,000 yuan, the urbanization rate is 72.57\%, and the proportion of urban basic medical insurance participation at the year-end is $91 \%$. Therefore, the increasing economic level, gradually increasing organizational management and social service security capabilities may be the main reasons for the star-like rise in the level of user system resilience. In September 2018, Qingdao municipal government issued "Qingdao water source construction and allocation" the 13th Five-Year Plan ", in which the water source construction projects mainly include reservoirs, pond DAMS and other storage projects and rainwater collection projects, recycled water utilization and seawater utilization and other water source and water supply projects; Water resources allocation engineering refers to the pipeline from water intake to water plant and supporting pumping station and other water transport infrastructure. The construction period of water supply and distribution pipeline is short. The input-output efficiency is high, and the improvement effect of system toughness ability is obvious, which is consistent with the change of toughness ability evaluation grade of water supply and distribution pipeline network. The toughness ability grade has increased from the medium level in 2018 to the high level in 2019. The construction period of the water source and water plant is long, and the actual benefits can only come into play after it is put into use and operation. Therefore, the toughness ability level of the water source and water plant has a longer growth period and a smaller improvement in the short term, which can be verified by combining with the evaluation results of 2018 and 2019. Although the resilience of users and water supply and distribution pipe network system has been improved in 2019, its contribution to the overall resilience level of the water supply and distribution system is limited, and the overall resilience of the water supply and distribution system in 2019 is still at a medium level. It is found that the low level of resilience of the water supply and distribution system is the main reason for this phenomenon, and the scarcity of water resources is the shortcoming of the overall resilience development of the water supply and distribution system in Qingdao. Improving water supply conditions and the construction of water sources is crucial for improving the resilience of the water supply and distribution system.

\section{Resilience ability improvement strategy}

Through comparative analysis, it can be seen that the main factors affecting the resilience of the water supply system can be summarized into four aspects ${ }^{[27-30]}$ : water source ecological conditions, economic development level, user water efficiency, and key water supply infrastructure. Therefore, the strategies to enhance the resilience of the urban water supply system will be discussed in the following part from the perspectives of the above four main factors.

(1) Abandon the development model of "pollution first, treatment later, treatment while pollution", and stick to the three development red lines of ecological function guarantee baseline, natural resource utilization limit, and ecological security bottom line.

(2) With the acceleration of urbanization, the demand for water in the suburbs and towns is gradually increasing, which poses a severe challenge to the integration of urban and rural water supply. Therefore, while ensuring the economic growth of urban areas, we should also increase the investment in the suburbs and towns, vigorously promote the new urbanization, so as to narrow the internal differences of the regional economy. 
(3) Implementing both water supply and water use control, improving water use efficiency, and making major water use efficiency indexes such as water consumption per 10,000 RMB of GDP and water consumption per 10,000 RMB of industrial added value reach the leading national and advanced international level.

(4) Adhere to the allocation of regional water sources, strengthen the construction of water supply network, strengthen the connection between municipal water supply backbone network and regional water supply network, enhance the toughness of water supply system, and ensure the safety of water supply in the whole region.

\section{Conclusion}

In this research, the assessment of resilience of urban water supply system under flood and drought disasters is studied.

Based on correlation analysis and factor analysis, the toughness evaluation index system of urban water supply system was constructed. The entropy weight method was used to determine the unified weight value of each index, and the comprehensive evaluation results of toughness of Qingdao water supply system based on cloud model were given. According to the evaluation, the overall resilience of the water supply system in Qingdao is at a medium level, and the main factors affecting the resilience are the ecological conditions of water sources, the economic development level, the efficiency of users' water use, and the key water supply infrastructure. In order to ensure the continuous improvement of the resilience of the urban water supply system, this paper discussed the resilience improvement strategy from the perspective of four main factors affecting the resilience of the water supply system in Qingdao, so as to provide decision support for further improving the resilience of the water supply system.

This study also has some shortcomings. The toughness model created in this paper is static, and the toughness of the water supply system is often its dynamic behavior in response to disturbance. Therefore, the dynamic evaluation of toughness under disturbance will be the focus of the next study.

\section{Data Availability}

414 No data is used to support this research.

\section{Conflicts of Interest}

The authors declare that there are no conflicts of interest regarding the publication of this paper.

\section{Color Processing / Printing}

We state that the figures are printed in color and are willing to pay an additional fee. 
All authors contributed equally to this work. All authors have read and agreed to the published version of the manuscript.

\section{Acknowledgements}

The authors would like to thank the National Natural Science Foundation of China (Grant No. 71471094, No. 71874123, No. 71704162), Humanities and Social Science Research Youth Fund Project of Ministry of Education (Grant No. 17YJC630184); Qingdao social science planning research project (Grant No. QDSKL1901192).

\section{Reference}

[1] Leah McMillan Polonenko, et al."Essential components of institutional and social indicators in assessing the sustainability and resilience of urban water systems: Challenges and opportunities." Science of the Total Environment 708.(2020): doi:10.1016/j.scitotenv.2019.135159.

[2] Puczko, K., Jekatierynczuk-Rudczyk, E. Extreme Hydro-Meteorological Events Influence to Water Quality of Small Rivers in Urban Area: A Case Study in Northeast Poland. Sci Rep 10, 10255(2020). https://doi.org/10.1038/s41598-020-67190-4

[3] Fiering M B. Alternative indices of resilience[J]. Water Resources Research, 1982, 18(1): 33-39.

[4] Hashimoto T, Stedinger J R, Loucks D P. Reliability, resiliency, and vulnerability criteria for water resource system performance evaluation[J]. Water resources research, 1982, 18(1): 14-20.

[5] Cuisong Y, Hao Z. Resilience classification research of water resources system in a changing environment[C]/2008 2nd International Conference on Bioinformatics and Biomedical Engineering. IEEE, 2008: 3741-3744.

[6] Tanner T, Mitchell T, Polack E, et al. Urban governance for adaptation: assessing climate change resilience in ten Asian cities[J]. IDS Working Papers, 2009, 2009(315): 01-47.

[7] Slobodan P. Simonovic. Systems Approach to Management of Water Resources-Toward Performance Based Water Resources Engineering[J]. Water, 2020, 12(4):1208.

[8] Jim W. Hall, Edoardo Borgomeo, Alexa Bruce, et al. Resilience of Water Resource Systems: Lessons from England[J]. Water Security, 2019, 8:100052.

[9] Kong J, Simonovic S P, Zhang C, et al. Resilience Assessment of Interdependent Infrastructure Systems: A Case Study Based on Different Response Strategies[J]. Sustainability, 2019, 11(23):6552.

[10] Pandit A, Crittenden J C. Index of network resilience (INR) for urban water distribution systems[J]. Nature, 2012, $12: 1-2$.

[11] David R, Pavel S, Simona S. Resilience of Critical Infrastructure Elements and Its Main Factors[J]. Systems, 2018, 6(2): 21.

[12] David R, Pavel S, Hromada M, et al. Complex approach to assessing resilience of critical infrastructure elements[J]. International Journal of Critical Infrastructure Protection, 2019, 25: 125-138.

[13] Zhao Sixiang, Liu Xiao, Zhuo Yihe, Fan Bo. Research on restoration strategy of the water supply system under sudden water pollution event [J]. Industrial Engineering and Management, 2015, 20(04):135-141. 
[14] Xu Tao, Wang Chunlian, Hong Min. Evaluation of resilience of urban waterlogging in China based on grey box model [J]. Urban Problems, 2015(04):2-11.

[15] Liu Jian, Huang Wenjie. A study on Evaluation of Elastic Capacity of Urban Water Supply System under the Influence of Salt Tide Scenarios [J]. Modern Urban Studies, 2017(09):32-40.

[16] Yu Kongjian, Xu Tao, Li Dihua, Wang Chunlian. Research Progress of Urban Water System Elasticity [J]. Urban Planning Forum, 2015(01):75-83.

[17] Li Qian, Guo Endong, Li Yuqin, Liu Zhibin. Analysis of key problems in seismic toughness evaluation of water supply system [J]. Journal of Catastrophology, 2019, 34(02):83-88.

[18] Behrooz Balaei, Suzanne Wilkinson, Regan Potangaroa, et al. Developing a Framework for Measuring Water Supply Resilience[J]. Natural Hazards Review, 2018, 19(4):04018013.

[19] Lukuba N. Sweya, Suzanne Wilkinson, Joseph Mayunga, et al. Development of a Tool to Measure Resilience against Floods for Water Supply Systems in Tanzania[J]. Journal of Management in Engineering, 2020, 36(4):05020007.

[20] Zhou Limin. Resilient Cities: Risk Management and Indicator Construction: An International Case Study [J]. Journal of Beijing Institute of Administration, 2016(02):13-20.

[21] Zhou Limin. Resilient Cities: Risk Management and Indicator Construction: An International Case Study [J]. Journal of Beijing Institute of Administration, 2016(02):13-20.

[22] Marks, S.J., Clair-Caliot, G., Taing, L. et al. Water supply and sanitation services in small towns in rural-urban transition zones: The case of Bushenyi-Ishaka Municipality, Uganda. npj Clean Water 3, 21 (2020). https://doi.org/10.1038/s41545-020-0068-4

[23] Li Deyi, Du Diao. Uncertainty Artificial Intelligence [M]. Beijing: National Defense Industry Press, 2005:137-153.

[24] He Jinping, Gao Quan, Shi Yuqun. Multi-level comprehensive evaluation method for dam safety based on cloud model [J]. Systems Engineering-Theory \& Practice, 2016, 36(11):2977-2983.

[25] Xu Zhaofeng, Tian Jiefang, Zhang Jing. Evaluation System and Optimization Strategy of Urban Resilience from the Perspective of Disaster Prevention [J]. China Safety Science Journal, 2019, 29(03): 1-7.

[26] Sun Honghu, Cheng Xianfu, Ni Ling, et al. Evaluation of flood control and disaster mitigation capacity of Chaohu Lake Basin based on cloud model and entropy weight method [J]. Journal of Catastrophology, 2015, 30(01):222-227.

[27] Bai Limin, Xiu Chunliang, Feng Xinghua, et al. Comprehensive assessment of urban resilience and its spatio-temporal differentiation in China [J]. World Regional Studies, 2019, 28(06):77-87.

[28] Li Ya, Zhai Guofang. Urban Disaster Resilience Assessment and Improvement Strategies in China. Planners, 2017, 33(08):5-11.

[29] Zhang Mingdou, Feng Xiaoqing. Comprehensive evaluation of urban resilience in China [J]. Urban Problems, 2018(10):27-36.

[30] LI Gang, XU Bo. Measurement and improvement path of urban resilience in China [J]. Journal of Shandong University of Science and Technology (Social Science Edition), 2018, 20(02): 83-89+116. 


\section{Supplementary Files}

This is a list of supplementary files associated with this preprint. Click to download.

- 5.Supplementarymaterial.doc 